\title{
FOXP3 and CTLA-4 genetic variants' influence on the susceptibility and clinical course of basal cell carcinoma
}

\author{
Martyna Sławińska, Monika Zabłotna, Roman J. Nowicki, Michał Sobjanek \\ Department of Dermatology, Venereology and Allergology, Medical University of Gdansk, Gdansk, Poland \\ Adv Dermatol Allergol 2021; XXXVIII (3): 455-460 \\ DOI: https://doi.org/10.5114/ada.2020.93368
}

\begin{abstract}
Introduction: The pathogenesis of basal cell carcinoma (BCC) is multifactorial and not fully elucidated. Previous studies showed that behaviour of the tumour may be influenced by the immune system and identified CD4+CD25 $5^{+}$FoxP3 ${ }^{+}$ regulatory $T$ cells $\left(T_{\text {regs }}\right.$ ) as dominant immune cells in BCC microenvironment. The function and development of Tregs is regulated by FOXP3, encoding transcription factor Forkhead box P3. FOXP3 regulates transcription of many genes, including up-regulation of cytotoxic lymphocyte-associated antigen-4 gene (CTLA-4). Expressed on Tregs, CTLA-4 interacts with antigen-presenting cells to inhibit T-cell activation.

Aim: To investigate the role of two polymorphisms (rs3761548 and rs2232365) of FOXP3 and CTLA-4 polymorphism (rs5742909) in BCC patients from northern Poland.

Material and methods: We analysed 280 unrelated patients with BCC of mean age $70.93 \pm 11.53(70.54 \pm 12.55$ women, $71.38 \pm 10.26$ men) and 200 healthy, unrelated age- and sex-matched volunteers.

Results: The differences in the occurrence of BCC between genotypes and alleles of the analysed polymorphisms were not statistically significant. In the studied group, the presence of the CC genotype in CTLA-4 rs5742909 polymorphism was statistically more frequent in patients with multiple BCCs.

Conclusions: It seems that the analysed FOXP3 and CTLA-4 polymorphisms do not influence the BCC susceptibility. CTLA-4 rs5742909 polymorphism may influence the susceptibility to multiple BCCs.
\end{abstract}

Key words: basal cell carcinoma, gene polymorphism, FOXP3, CTLA-4.

\section{Introduction}

The pathogenesis of basal cell carcinoma (BCC) is multifactorial and not fully elucidated. Previous studies showed that behaviour of the tumour may be influenced by the immune system and identified $\mathrm{CD} 4^{+} \mathrm{CD} 25^{+} \mathrm{FoxP} 3^{+}$regulatory $T$ cells $\left(T_{\text {regs }}\right)$ as dominant immune cells in BCC microenvironment [1-3]. It is known that different types of human cancer are able to recruit immune cells to the tumour site to promote escape from host immune surveillance. After recruitment from peripheral blood, $T_{\text {regs }}$ play a key role in the suppression of anti-tumour immunity [4]. The function and development of $\mathrm{T}_{\text {regs }}$ is regulated by FOXP3 encoding transcription factor, Forkhead box P3 (FoxP3). FoxP3 regulates transcription of many genes, including up-regulation of cytotoxic lymphocyte-associated antigen-4 gene (CTLA-4) [5]. Expressed on $\mathrm{T}_{\text {regs }}$, CTLA-4 interacts with antigen-presenting cells to inhibit T-cell activation [6].

Polymorphisms of FOXP3 and CTLA-4 may change their role functionally or quantitatively, therefore leading to an altered function of $\mathrm{T}_{\text {regs }}$ [7]. The role of single nucleotide polymorphisms (SNPS) of these genes has been previously investigated in different human neoplasia [8, 9]. To our knowledge, none of the previous studies investigated the role of FOXP3 polymorphisms in BCC, while only one study concerned the role of CTLA-4 variants in the tumour development [6].

\section{Aim}

The aim of the study was to investigate the role of two polymorphisms (rs3761548 and rs2232365) of FOXP3 and CTLA-4 polymorphism (rs5742909) in BCC patients from northern Poland.

\section{Material and methods \\ Patients and controls \\ The study included 280 unrelated patients with BCC of mean age $70.93 \pm 11.53(70.54 \pm 12.55$ women, 71.38}

Address for correspondence: Martyna Sławińska MD, Department of Dermatology, Venereology and Allergology, Medical University of Gdansk, 17 Smoluchowskiego St, 80-214, Gdansk, Poland, phone: +48 5858440 14, e-mail: mslawinska@gumed.edu.pl Received: 9.12.2019, accepted: 27.01.2020. 
\pm 10.26 men) and 200 healthy, unrelated age- and sexmatched volunteers. Clinical characteristics of the study group are presented in Table 1. Patients were treated due to primary or recurrent BCC in the Department of Dermatology, Venereology and Allergology, Medical University of Gdansk, Poland. Histopathological diagnoses were made based on the assessment of the haematoxylin and eosin stained sections, according to the classification system of the World Health Organization (WHO) [10]. SNPs were chosen based on their previously documented significance in other neoplasia, on their functional relevance, as well as on the minor allele frequency MAF $\geq 0.1$ in the Caucasian population (https:// www.ncbi.nlm.nih.gov/snp/). Clinical data were collected based on medical records. None of the subjects were organ transplant recipients, none were being treated with immunosuppressive drugs, none suffered from any systemic inflammatory disease or malignancy, none had the signs of infection. The blood samples were collected after obtaining patients' informed consent, before the surgical procedure. The status of "multiple BCC" was assigned based on previous medical records, medical history or more than one tumour detected during physical examination on hospital admission. Patients with multiple tumours were evaluated for the clinical and radiological signs of Gorlin-Goltz syndrome and, if necessary, referred to further genetic assessment. Patients with confirmed diagnosis of Gorlin-Goltz syndrome were excluded from the study. Skin surface in anatomical regions considered as chronically exposed to sunlight, namely the face, top of the ears, back of the neck, dorsum of the hands and forearms was classified as "area exposed to UV". All subjects were exclusively of Polish descent. The study was approved by the local research ethics committee of the Medical University of Gdańsk.

\section{CTLA-4 and FOXP3 genotyping}

DNA from the peripheral blood of BCC patients and volunteers was isolated by Blood Mini A\&A Biotechnology (A\&A Biotechnology, Gdańsk, Poland). FOXP3 rs3761548 and rs2232365 polymorphisms and CTLA-4 rs5742909 polymorphism were analysed using polymerase chain reaction with sequence-specific primers (SSP-PCR) [11, 12].

\section{Ethical disclosure}

The study was approved by the local ethics committee of the Medical University of Gdansk.

\section{Statistical analysis}

The $\chi^{2}$ analysis was used to compare the observed number of genotypes with that expected for a population in a Hardy-Weinberg equilibrium. The $\chi^{2}$ analysis was also employed to test the significance of the differences in the observed alleles and genotypes between groups. The Mann-Whitney $U$ test was used to compare the mean values. Due to the fact that FOXP3 is located on the X-chromosome, the analysis was stratified for gender. The strength of associations between rs3761548 and rs2232365 FOXP3 polymorphisms and

Table 1. Characteristics of the study group - BCC patients

\begin{tabular}{|c|c|c|c|}
\hline Parameter & Males & Females & Overall group \\
\hline$N(\%)$ & $132(47.1)$ & $148(52.9)$ & 280 \\
\hline \multicolumn{4}{|l|}{ Age, mean \pm SD [years]: } \\
\hline$<60$ & $54.95 \pm 5.12$ & $52.32 \pm 5.33$ & $53.22 \pm 5.37$ \\
\hline$>60$ & $74.29 \pm 7.92$ & $76.52 \pm 7.40$ & $75.42 \pm 7.73$ \\
\hline Tumour size $[\mathrm{cm}]$ & $1.37 \pm 0.84$ & $2.18 \pm 8.85$ & $1.8 \pm 6.47$ \\
\hline \multicolumn{4}{|l|}{ Diagnosis: } \\
\hline $\mathrm{BCC}$ & $125(47.7 \%)$ & $137(52.3 \%)$ & 262 \\
\hline $\mathrm{BCC}$ recurrence & $7(38.9 \%)$ & $11(61.1 \%)$ & 18 \\
\hline \multicolumn{4}{|l|}{ Number of tumours: } \\
\hline One tumour & $74(41.1 \%)$ & $106(58.9 \%)$ & 180 \\
\hline Multiple tumours & $58(58.0 \%)$ & $42(42.0 \%)$ & 100 \\
\hline \multicolumn{4}{|l|}{ Location: } \\
\hline Area exposed to UV & $105(44.9 \%)$ & $129(55.1 \%)$ & 234 \\
\hline Area not exposed to UV & $27(58.7 \%)$ & $19(41.3 \%)$ & 46 \\
\hline
\end{tabular}


rs5742909 CTLA-4 polymorphism and BCC risk was assessed in the allelic model, as well as the genotype recessive model, dominant model, and overdominant model (Tables 2, 3).

Analyses were performed using the Statistica 12.0 software package (StatSoft, Inc., 2010). $P \leq 0.05$ was considered statistically significant.

\section{Results}

\section{FOXP3 polymorphisms}

Table 2 shows genotype and allele frequencies for FOXP3 rs3761548 and FOXP3 rs2232365 polymorphisms in BCC patients and controls. Due to the fact that FOXP3 is located on the X-chromosome, the analy- sis was stratified for gender. The deviation of genotype distribution from Hardy-Weinberg equilibrium was analysed only in women (males carry only one X-chromosome). The distribution of the FOXP3 rs3761548 and FOXP3 rs2232365 in females was consistent with the Hardy-Weinberg equilibrium in the control group and inconsistent within the females from the studied group. No association was observed between SNP alleles and genotypes and BCC risk. In the BCC group there were no statistically significant associations between FOXP3 rs3761548 as well as FOXP3 rs2232365 allele and genotype frequencies and tumour size, location, and number of tumours diagnosed. We have found no statistical differences between the group of patients with primary and recurrent BCC.

Table 2. Genotypes and alleles frequencies for FOXP3 rs3761548 and rs2232365 in BCC patients and controls

\begin{tabular}{|c|c|c|c|c|c|}
\hline \multirow[t]{2}{*}{ SNP } & \multicolumn{2}{|c|}{ BCC group } & \multicolumn{2}{|c|}{ Control group } & \multirow[t]{2}{*}{$P$-value } \\
\hline & Females & Males & Females & Males & \\
\hline \multicolumn{6}{|l|}{ rs3761548: } \\
\hline \multicolumn{5}{|c|}{ Recessive model: } & \multirow[t]{3}{*}{0.97} \\
\hline AA & 34 (23.0\%) & & $23(22.8 \%)$ & & \\
\hline$C A+C C$ & $114(77.0 \%)$ & & 78 (77.2\%) & & \\
\hline \multicolumn{5}{|c|}{ Dominant model: } & \multirow[t]{3}{*}{0.17} \\
\hline$A A+C A$ & 87 (58.8\%) & & $68(67.3 \%)$ & & \\
\hline $\mathrm{CC}$ & 61 (41.2\%) & & 33 (32.7\%) & & \\
\hline \multicolumn{5}{|c|}{ Overdominant model: } & \multirow[t]{3}{*}{0.16} \\
\hline CA & $53(35.8 \%)$ & & 45 (44.5\%) & & \\
\hline$C C+A A$ & 95 (64.2\%) & & $56(55.5 \%)$ & & \\
\hline C & & $63(47.7 \%)$ & & $54(54.6 \%)$ & 0.31 \\
\hline A & & 69 (52.3\%) & & 45 (45.4\%) & \\
\hline \multicolumn{6}{|l|}{ rs2232365: } \\
\hline \multicolumn{5}{|c|}{ Recessive model: } & \multirow[t]{3}{*}{0.25} \\
\hline AA & $56(37.8 \%)$ & & $31(30.7 \%)$ & & \\
\hline$A G+G G$ & $92(62.2 \%)$ & & 70 (69.3\%) & & \\
\hline \multicolumn{5}{|c|}{ Dominant model: } & \multirow[t]{3}{*}{0.53} \\
\hline$A A+A G$ & $100(67.6 \%)$ & & 72 (71.3\%) & & \\
\hline GG & $48(32.4 \%)$ & & $29(28.7 \%)$ & & \\
\hline \multicolumn{5}{|c|}{ Overdominant model: } & \multirow[t]{3}{*}{0.07} \\
\hline AG & $44(29.7 \%)$ & & $41(40.6 \%)$ & & \\
\hline$A A+G G$ & $104(70.3 \%)$ & & 60 (59.4\%) & & \\
\hline \multicolumn{2}{|l|}{$A$} & $68(51.5 \%)$ & & $52(52.5 \%)$ & \multirow[t]{2}{*}{0.88} \\
\hline G & & 64 (48.5\%) & & 47 (47.5\%) & \\
\hline
\end{tabular}


Table 3. Genotype and allele frequencies for CTLA-4 rs5742909 in BCC patients and controls

\begin{tabular}{lccc}
\hline Genotypes and alleles & BCC group & Control group & $P$-value \\
\hline Recessive model: & & & 0.33 \\
\hline $\mathrm{TT}$ & $6(2.1 \%)$ & $2(1.0 \%)$ & \\
\hline $\mathrm{CT}+\mathrm{CC}$ & $274(97.9 \%)$ & $198(99.0 \%)$ & 0.78 \\
\hline Dominant model: & & $38(19.0 \%)$ & 0.97 \\
\hline $\mathrm{CT}+\mathrm{CT}$ & $56(20.0 \%)$ & $162(81.0 \%)$ & \\
\hline Overdominant model: & $224(80.0 \%)$ & & \\
\hline $\mathrm{CT}$ & & $36(18.0 \%)$ & 0.59 \\
\hline $\mathrm{C}+\mathrm{TT}$ & $50(17.9 \%)$ & $164(82.0 \%)$ & \\
\hline $\mathrm{T}$ & $230(82.1 \%$ & $360(90.0 \%)$ & \\
\hline
\end{tabular}

\section{CTLA-4 polymorphisms}

Table 3 shows genotype and allele frequencies for CTLA-4 rs5742909 polymorphisms in BCC patients and controls. The distribution of the CTLA-4 genotypes was consistent with the Hardy-Weinberg equilibrium both in BCC patients and controls. No association was observed between SNP alleles and genotypes and BCC risk. The presence of the CC genotype in CTLA-4 rs5742909 polymorphism was statistically more frequent in patients with multiple BCCs $(p=0.05)$. There were no statistically significant associations between CTLA-4 rs5742909 allele and genotype frequencies and tumour size, and location. We have found no statistical differences between the group of patients with primary and recurrent BCC.

\section{Discussion}

$T_{\text {regs }}$ have been previously recognized as one of the key elements modulating anti-tumour immunity and involved in the pathogenesis of numerous human neoplasia [1-3]. The population of $\mathrm{T}_{\text {regs, }}$ which constitutes about $5 \%$ of CD4+ T lymphocytes, is characterized by high-intensity expression of CD25 and involves two main subsets [13]. Naturally occurring $T_{\text {regs }}$ (nTregs) originating in the thymus during the process of negative selection are responsible for maintaining peripheral tolerance. Inducible or adaptive $T_{\text {regs }}\left(\mathrm{iT}_{\text {regs }}\right.$, Tr1) are induced in the periphery, where features necessary for controlling local immune response are acquired [4]. The latter are known to possess a broader range of suppressor properties, what concerns especially $\mathrm{iT}_{\text {regs }}$ isolated from human tumour microenvironment [14]. Initial studies showed that accumulation of $\mathrm{T}_{\text {regs }}$ in the tumour site and peripheral blood suppresses anti-tumour response, leading to cancer progression and therefore has been recognized as a marker of poor prognosis. However, recent studies showed that presence of $T_{\text {regs }}$ may also improve prognosis in several inflammation-associated neoplasia [4, 15]. Inhibition of anti-tumour immune response by $T_{\text {regs }}$ is complex and involves several mechanisms like production of suppressive cytokines (IL-10, TGF- $\beta$, IL-35), direct cytotoxicity (via perforins/granzyme), immune checkpoints/inhibitory receptors (CTLA-4, PD-1, IDO, TIM-3, ICOS, TIGIT, IDO) and induction of tolerogenic dendritic cells [16]. Development and functioning of $T_{\text {regs }}$ is primarily regulated by the FOXP3 gene, encoding FOXP3 transcription factor, located at the small arm of the X-chromosome (Xp11.23). FOXP3 regulates transcription of numerous target genes, including up-regulation of CTLA-4 (encoding CTLA-4 receptor). CTLA-4 is a molecule constitutively expressed in $\mathrm{T}_{\text {regs }}$ and critical for their suppressive function. According to Takeuchi and Nishikawa [17], main mechanisms mediated by CTLA-4 involve blocking of B7-CD28 signals through binding to B7 with higher avidity and antigen presenting cells maturation restriction.

Although it is known that $\mathrm{CD} 4+\mathrm{CD} 25+\mathrm{FoxP3}+$ regulatory $T$ cells are highly prevalent in BCC microenvironment, their role in the tumour pathogenesis is underinvestigated [1-3]. Chen et al. [18] who examined the effects of UV exposure on FOXP3+ peri-neoplasm cells infiltration in non-melanoma skin cancer (NMSC) have found that they were statistically less prevalent in more pigmented BCCs. The authors hypothesised that pigment is responsible for UV radiation (UVR) obstruction, and thus inhibits the induction of FOXP3+cells. One of the possible pathogenetic pathways that could play a role in UVR-induced FOXP3 expression includes the UVR DNA damage that leads to p53 pathway activation [19]. Previous studies provided also evidence on the role of CTLA-4 in UVR immunosuppression in skin cancer development [6, 20, 21]. 
Beissert et al. [21] have shown that transgenic mice expressing CTLA-4 antagonist, CTLA-4lg, developed fewer skin tumours after chronic UVR exposure, compared to their wild-type counterparts. Studies exploring the role of $T_{\text {regs }}$ in BCC provide also important knowledge on its nonsurgical treatment methods mechanisms. Imiquimod, a topical drug approved for the treatment of superficial BCC, is a Toll-like receptor 7 agonist which exerts an antitumour effect via modification of the immune response, which is associated with the reduction in $T_{\text {regs }}$ population in BCC environment [22]. Nevertheless, the drug may also exert counteracting immunosuppressive effect via inducing $T_{\text {regs }}$, which may influence its unpredictable treatment effect in thicker tumours [23]. Nomikos et al. [22] have shown that application of cryosurgery during imiquimod treatment (immunocryosurgery) significantly decreases $T_{\text {regs }}$ infiltration, exerting local anti-tumour response. The role of CTLA-4 in BCC may also have a therapeutic significance. Mohan et al. [24] reported a regression of advanced BCC in a patient treated with ipilimumab (anti-CTLA-4 monoclonal antibody) due to concurrent metastatic melanoma. This case report suggests that anti-CTLA-4 antibodies could potentially be used in the treatment of BCC, however further studies are needed to confirm this observation.

A series of FOXP3 polymorphisms located in the promoter, introns and exons have been described [25]. The promoter region polymorphism may influence gene expression and subsequently $T_{\text {regs }}$ function through changing the kinetics of transcription initiation or changing transcription factors binding specificity [7]. In the present study we focused on the significance of two FOXP3 promoter SNPs: -3279 C/A (rs3761548) and -924 A/G (rs2232365) in BCC patients. The role of -3279 C/A (rs3761548) FOXP3 polymorphism has been previously associated with several neoplasia. The presence of A allele correlated with a higher risk of thyroid cancer, non-small cell lung cancer, colorectal cancer, breast cancer, ovarian cancer and Wilms' tumour [8, 26-30]. To our knowledge only one study examined the correlation between rs3761548 SNP and FOXP3 expression in neoplastic tissue [28]. Lopes et al. [28] found that the presence of -3279 A allele in breast cancer patients correlated with increased (but not statistically significant) expression of FOXP3. The role of $-924 \mathrm{~A} / \mathrm{G}$ (rs2232365) polymorphism has been previously examined in patients with Wilms' tumour and breast cancer [30, 31]. In both studies the presence of -924 A allele was associated with higher disease susceptibility. Previously authors noticed that rs2232365 SNP has been located in a presumed binding site of GATA-3 transcription factor, which is able to bind to the promoter and induce FOXP3 expression only in the presence of A allele [30, 32]. To the best of our knowledge, none of the previous studies examined the association between the above mentioned FOXP3 polymorphisms and BCC susceptibility. In the present study no association was observed between examined FOXP3 polymorphisms and BCC risk.

CTLA-4 -318C/T (rs5742909) polymorphism is located within the promoter region of CTLA-4 gene (2q33.2). Previous studies showed that the presence of $-318 \mathrm{~T}$ allele is associated with higher promoter activity and increased surface CTLA-4 expression [33, 34]. Therefore it has been hypothesized that the presence of $-318 \mathrm{~T}$ allele may enhance $T_{\text {regs }}$ suppressor activity, subsequently increasing the risk of cancer development/progression. Meta-analysis performed by Zhang et al. [9] showed that the presence of T allele was associated with an increased cancer risk among Europeans, but not among Asians. To our knowledge, only one study examined the role of CTLA-4 CT60 polymorphism in BCC susceptibility [6]. The role of -318C/T (rs5742909) polymorphism in this neoplasia has not been investigated so far.

In the present study we have found no association between CTLA-4 rs5742909 polymorphism and BCC susceptibility, however, the presence of CC genotype was statistically more frequent in patients with multiple BCCs.

There are several limitations concerning the study. First, the studied population was selected from one region, so the results may not be representative for other populations. Secondly, the studied group was relatively small, which could influence the analysis statistical power. The third limitation was the retrospective collection of some data, like the previous history of BCC, as not all patients had complete medical records/good knowledge about previously excised skin lesions. As it was difficult to objectively measure the lifetime exposure to sunlight we had decided to consider sun-exposed vs. non-sunexposed skin areas instead.

\section{Acknowledgments}

The study was funded by the Medical University of Gdansk, Project No. 02-0066/07/253.

\section{Conflict of interest}

The authors declare no conflict of interest.

\section{References}

1. Rangwala S, Tsai KY. Roles of the immune system in skin cancer. Br J Dermatol 2011; 165: 953-65.

2. Kaporis HG, Guttman-Yassky E, Lowes MA, et al. Human basal cell carcinoma is associated with Foxp3+ T cells in a Th2 dominant microenvironment. J Invest Dermatol 2007; 127: 2391-8.

3. Omland SH, Nielsen PS, Gjerdrum LMR, Gniadecki R. Immunosuppressive environment in basal cell carcinoma: the role of regulatory T cells. Acta Derm Venereol 2016; 96: 917-21.

4. Whiteside TL. What are regulatory T cells (Treg) regulating in cancer and why? Semin Cancer Biol 2012; 22: 327-34.

5. Szylberg L, Karbownik D, Marszalek A. The role of FOXP3 in human cancers. Anticancer Res 2016; 36: 3789-94. 
6. Welsh MM, Applebaum KM, Spencer SK, et al. CTLA4 variants, UV-induced tolerance, and risk of non-melanoma skin cancer. Cancer Res 2009; 69: 6158-63.

7. Oda JMM, Hirata BKB, Guembarovski RL, Watanabe MAE. Genetic polymorphism in FOXP3 gene: imbalance in regulatory T-cell role and development of human diseases. J Genet 2013; 92: 16-171.

8. Jiang W, Zheng L, Xu L, et al. Association between FOXP3 gene polymorphisms and risk of differentiated thyroid cancer in Chinese Han population. J Clin Lab Anal 2017; 31: doi: 10.1002/jcla.22104.

9. Zhang Y, Zhang J, Deng Y, et al. Polymorphisms in the cytotoxic T-lymphocyte antigen 4 gene and cancer risk: a metaanalysis. Cancer 2011; 117: 4312-24.

10. LeBoit PE, Burg G, Weedon D, Sarasin A. Skin Tumors: Pathology and Genetics. International Agency for Research on Cancer Press. Lyon, France 2006

11. Gao L, Li K, Li F, et al. Polymorphisms in the FOXP3 gene in Han Chinese psoriasis patients. J Dermatol Sci 2010; 57 : 51-6.

12. Kusztal M, Kościelska-Kasprzak K, Drulis-Fajdasz D, et al. The influence of CTLA-4 gene polymorphism on long-term kidney allograft function in Caucasian recipients. Transpl Immunol 2010; 23: 121-4.

13. Frydrychowicz M, Boruczkowski M, Kolecka-Bednarczyk A, Dworacki G. The dual role of Treg in cancer. Scand J Immunol 2017; 86: 436-43.

14. Strauss L, Bergmann C, Szczepanski M, et al. A unique subset of CD4+CD25highFoxp3+ T cells secreting interleukin-10 and transforming growth factor-beta1 mediates suppression in the tumor microenvironment. Clin Cancer Res 2007; 13: 4345-54.

15. Shang B, Liu Y, Jiang SJ, Liu Y. Prognostic value of tumorinfiltrating FoxP3+ regulatory T cells in cancers: a systematic review and meta-analysis. Sci Rep 2015; 5: 15179.

16. Chaudhary B, Elkord E. Regulatory T cells in the tumor microenvironment and cancer progression: role and therapeutic targeting. Vaccines 2016; 4: E28.

17. Takeuchi Y, Nishikawa H. Roles of regulatory $T$ cells in cancer immunity. Int. Immunol 2016; 28: 401-9.

18. Chen L, Shen Z, Wu J. Effects of ultraviolet radiation exposure on FOXP3+ infiltration in cutaneous squamous cell carcinoma and basal cell carcinoma. Photodermatol Photoimmunol Photomed 2011; 27: 297-303.

19. Jung DJ, Jin DH, Hong SW, et al. Foxp3 expression in p53dependent DNA damage responses. J Biol Chem 2010; 285: 7995-8002.

20. Schwarz A, Beissert S, Grosse-Heitmeyer K, et al. Evidence for functional relevance of CTLA-4 in ultraviolet-radiationinduced tolerance. J Immunol 2000; 165: 1824-31.

21. Beissert S, Bluestone JA, Mindt I, et al. Reduced ultravioletinduced carcinogenesis in mice with a functional disruption in B7-mediated costimulation. J Immunol 1999; 163: 6725-31.

22. Nomikos K, Lampri E, Spyridonos P, Bassukas ID. Alterations in the inflammatory cells infiltrating basal cell carcinomas during immunocryosurgery. Arch Dermatol Res 2019; 311: 499-504.

23. Conroy H, Marshall NA, Mills KH. TLR ligand suppression or enhancement of Treg cells? A double-edged sword in immunity to tumours. Oncogene 2008; 27: 168-80.

24. Mohan SV, Kuo KY, Chang ALS. Incidental regression of an advanced basal cell carcinoma after ipilimumab exposure for metastatic melanoma. JAAD Case Reports 2016; 2: 13-5.

25. Nedoszytko B, Lange M, Sokołowska-Wojdyło M, et al. The role of regulatory $T$ cells and genes involved in their differ- entiation in pathogenesis of selected inflammatory and neoplastic skin diseases. Part II: The Treg role in skin diseases pathogenesis. Adv Dermatol Allergol 2017; 34: 405-17.

26. He YQ, Bo Q, Yong W, et al. FoxP3 genetic variants and risk of non-small cell lung cancer in the Chinese Han population. Gene 2013; 531: 422-5.

27. Chen L, Yu Q, Liu B, Zhu L. Association of FoxP3 rs3761548 polymorphism with susceptibility to colorectal cancer in the Chinese population. Med Oncol 2014; 31: 374.

28. Lopes LF, Guembarovski RL, Guembarovski AL, et al. FOXP3 transcription factor: a candidate marker for susceptibility and prognosis in triple negative breast cancer. Biomed Res Int 2014; 2014: 341654.

29. Zhang Y, Xu L, Zhou B, et al. Transcription factor FOXP3 gene variants affect epithelial ovarian carcinoma in the Han Chinese population. Int J Clin Exp Pathol 2018; 11: 1684-93.

30. Ozawa PMM, Ariza CB, Losi-Guembarovski R, et al. Wilms' tumor susceptibility: possible involvement of FOXP3 and CXCL12 genes. Mol Cell Pediatr 2016; 3. http://dx.doi. org/10.1186/s40348-016-0064-4. Accessed August 1, 2019.

31. Banin Hirata BK, Losi Guembarovski R, Vitiello GAF, et al. FOXP3 allelic variants and haplotype structures are associated with aggressive breast cancer subtypes. Dis Markers 2017; 2017: 6359603.

32. You Z, Wu Z, Zhang C, et al. Association between functional polymorphisms of Foxp3 gene and the occurrence of unexplained recurrent spontaneous abortion in a Chinese Han population. Clin Dev Immunol 2012; 2012: 896458.

33. Wang XB, Zhao X, Giscombe R, Lefvert AK. A CTLA-4 gene polymorphism at position -318 in the promoter region affects the expression of protein. Genes Immun 2002; 3: 233-4.

34. Ligers A, Teleshova N, Masterman T, et al. CTLA-4 gene expression is influenced by promoter and exon 1 polymorphisms. Genes Immun 2001; 2: 145-52. 